\title{
Ontology for relativistic collapse theories
}

Wayne C. Myrvold

Department of Philosophy

The University of Western Ontario

Forthcoming in Olimpia Lombardi, Sebastian Fortin, Cristian López and Federico Holik, eds., Quantum Worlds (Cambridge University Press).

\section{Introduction}

Dynamical collapse theories, such as the GRW theory (Ghirardi, Rimini and Weber 1986), the Continuous Spontaneous Localization theory, or CSL (Pearle 1989; Ghirardi, Pearle and Rimini 1990), Quantum Mechanics with Universal Spontaneous Localization, or QMUPL (Diósi 1989), and their respective relativistic extensions (Dove 1996; Dove and Squires 1996; Tumulka 2006; Bedingham 2011a,b; Pearle 2015) modify the usual deterministic, unitary quantum dynamics in such as to produce something like the textbook collapse process. See Bassi and Ghirardi (2003), Bassi et al. (2013), and Ghirardi (2016) for overviews.

If some sort of dynamical collapse theory is correct, what might the world be like? Can a theory of that sort be a quantum state monist theory, or must such theories supplement the quantum state ontology with additional beables? In a previous work (Myrvold 2018), I defended quantum state monism. The view defended involves a natural extension of the usual eigenstate-eigenvalue link, which provides a sufficient condition for a quantum state to be one in which a system has a definite value of some dynamical variable, namely, that the quantum state be an eigenstate of that variable. The usual eigenstate-eigenvalue link leaves it open what to say about states that are not eigenstates. A state that is not an eigenstate of some dynamical variable, but is very close to an eigenstate, exhibits behaviour that closely approximates that of the eigenstate. In accordance with a proposal of Ghirardi, Grassi, and Pearle (1990), in such a case the quantity may be treated as if it were definite. But specification of the quantities that are definite or near-definite does not exhaustively specify the condition of the physical world, as there are matters of fact about such things as the spread of values of a dynamical variable in a given state. The natural ontology for a collapse theory is a distributional ontology along the lines advocated by Philip Pearle (2009). On such an account, dynamical quantities such as charge or mass within a specified region do not take on precise values, but, rather, have associated with them a distribution of values. 
This chapter discusses the extension of such a picture to the context of a relativistic spacetime. This will not be presumed to be Minkowski spacetime, as we will want to consider curved spacetimes; furthermore, we do not wish to exclude the possibility of discrete spacetimes. What the spacetimes we will consider have in common is a relativistic causal structure. In particular, we will focus attention on spacetimes in which, for any spacetime point $p$, there are temporally extended processes that go on at spacelike separation from $p$, prohibited by the spacetime structure from either causally influencing $p$ or being influenced by it.

In Section 2 I give a brief recapitulation of the argument, presented in more detail in Myrvold (2018), for distributional ontology. This is based on a principle that, I claim, ought to be respected by any project of seeking to draw ontological conclusions from non-fundamental physical theories, a principle that I call the Principle of Metaphysical Continuity, outlined in section 2.1. This principle permits us to draw conclusions about ontology for non-ideal collapse theories - that is, collapse theories that yield, not exact eigenstates of the dynamical quantities one would like to be definite, but close approximations to them-from ontological considerations regarding ideal collapse theories. In Section 3 is presented a fairly general schema for collapse theories in a relativistic spacetime. Finally, in section 4, we turn to the identification of local beables for theories of that sort.

\section{The case for distributional ontology}

\subsection{Ontology for non-fundamental theories}

If we want to know something about the make-up of the world, we can do no better than to look to our best scientific theories. This poses a prima facie problem, however, as there is not, and never has been, a convincing candidate on the table for a complete and fundamental physical theory.

One reaction to this fact might be take on the task of giving an account of what the world would be like if such-and-such physical theory were a complete and fundamental theory. On this view, metaphysics is a subgenre of fiction, though stripped of plot and character and, indeed, of everything that motivates us to read fiction. This strikes me as an uninteresting enterprise, except insofar as considerations of unrealistic theories yields insights regarding the ontology of the actual world. For instance: though we currently lack a theory that incorporates both quantum and gravitational phenomena, one could, perhaps, investigate the structure of a world in which there is 
no gravitation and in which the standard model of quantum field theory is exactly correct. But such a world would be a lonely and boring place, as it would contain no stars or planets, and, since virtually all nuclei heavier than helium are formed in stars, would contain little in the way of chemical reactions. We could not ask what it would like to be a denizen of a world like that, because a world like that would contain no life.

Another reaction might be to abandon all ontological inquiry as hopeless, on the grounds that we can expect future theories to generate radical ontological shifts. This strikes me as overly pessimistic. The objects dealt with in classical physics do, after all, exist, even if their behaviour is not exactly what classical physics would lead one to expect. Any theory that can lay claim to the title of a viable successor theory to our current theories is obliged to recover the empirical successes of our current theories, and, as long as we resist the temptation to draw stronger conclusions from our current physical theories than we have warrant for, there are reasons for optimism that those conclusions will weather the storms of future theory change.

This sort of attitude recommends due caution in our metaphysical musings. The evidence we have concerning our physical theories warrants only the conclusions that they hold to a good approximation in their domains of applicability, and that any viable successor theory will have entail something like the current theories within those domains. If our current theories have metaphysical consequences that are sensitive to the precise details of the theory, consequences that would not hold if the theory were slightly different, then we have no warrant for taking those consequences to hold of our world. Our metaphysical conclusions should satisfy a Principle of Metaphysical Continuity: they should be robust under small perturbations of theory. This is a principle that we will put to work, in Subsection 2.3, below.

\subsection{The requirement of local beables}

Consider a region of spacetime that is bounded in both time and space, say, the spatial region inside your office, during some specified hour of time. Of the things that are true of that bounded spacetime region, some are local to that region: they refer only to intrinsic properties of that region. These are to be contrasted with things that involve relations to states of affairs outside the region, or either implicit or explicit reference to things outside. 
For example, on the usual way of thinking about things, if your office, during the hour we are considering, contains a cabinet-shaped piece of steel, this is a local fact about that spacetime region. If the proposition that the spacetime region under consideration contains an object of that sort is true, its truth is compatible with completely arbitrary states of affairs outside the region, and its truth value cannot be changed by goings-on outside the region unless those goings-on have an effect on local matters within the region. By contrast, if the contents of your office are approximately 150 million kilometers from the nearest star, this fact is clearly a fact about relations between the things in your office and the world outside of it. A symptom of this fact is that it can be changed by making changes outside your office that don't affect anything within it.

By a local beable, I will mean something that is, in this sense, local to a bounded spacetime region. The ontology of a physical theory might contain both local and nonlocal beables. If it is the case that, for an arbitrarily fine covering of spacetime with open sets, the full ontology of the theory supervenes on beables that are local to elements of that covering, we will say that the ontology satisfies the condition of separability (see Myrvold 2011 for further discussion).

Quantum state realism entails rejection of separability. It does not follow that there are no local beables. For one thing, there could be local beables postulated in addition to the quantum state. But also, some aspects of the quantum state - in particular, the reduced state that is the restriction of the state to observables pertaining to a bounded spacetime region-might be counted as local beables.

Need there be any local beables at all? If we are willing to countenance a rejection of separability, might we not go all the way, and accept a radically holistic view in which there are no beables intrinsic to any region short of the whole of spacetime?

The difficulty with this is that, if the theory is meant to be one that is in principle comprehensive, it must have room for such things as experimental apparatus that is subject to local manipulations, and whose experimental readouts are, presumably, matters of fact local to the laboratory. In the absence of things like these, the theory runs the risk of undermining its own evidential base (see Maudlin 2007 for a lucid discussion of this point).

A brief comment, before we continue. What it means to say that a structure found within a physical theory plays the role of spacetime for that theory is that it has the appropriate connections with dynamics. In speaking of spacetime, I will always mean that structure that plays the role in 
the theory of affording spatiotemporal relations such as distances, temporal intervals, causal connectability, and the like, distances and temporal intervals and causal relations that are relevant to the dynamics. It is necessary to say this because it has been claimed that quantum theory motivates the introduction of a so-called "fundamental space" or "fundamental arena," a highdimensional space that would be such that quantum states involve nothing more than assignments of local beables to points in that fundamental space (see Albert 1996 and the various contributions to Ney and Albert, eds., 2013). In a quantum theory, even if such a space can be found, it is not the structure on which the distances, temporal intervals, and causal relations relevant to the dynamics are defined. For that reason, such a space, even if it were to exist, is not spacetime in the sense of the word being used in this chapter. Hence, even if such a space did exist, a quantum state realist ontology violates separability, as we are using the term.

\subsection{Ontology for ideal collapse theories}

According to the textbook collapse postulate, after an experiment the quantum state of the system subjected to the experiment is an eigenstate of the observable whose value has been obtained. Naively, one might expect a dynamical collapse theory to be like that. There are good reasons for thinking that this is an unattainable goal. If, however, we could have a theory like that - a theory that yielded eigenstates of an appropriate dynamical variable - then, I claim, there would be no problem of ontology for the theory, once we have settled on a choice of dynamical variable to collapse to eigenstates of (a suitable choice seems to be that of a smeared mass density, as advocated by Ghirardi, Grassi, and Benatti 1995). That there is any question about the ontology for a collapse theory is an aspect of what has been called the tails problem (first flagged as an issue by Shimony 1991, and by Albert and Lower 1991), which stems from the fact that collapse theories do not lead to eigenstates of familiar dynamical quantities.

Consider a quantum theory on a discrete spacetime, one on which space consists of elementary cells of size vastly smaller than the scales on which we deal with things. Suppose we had a collapse theory that tended to suppress superpositions of distinct mass densities smeared over regions (which could consist of a great many of these elementary cells) of order $10^{-5} \mathrm{~cm}$, small on human scale, but large compared to atomic dimensions. Suppose that our collapse theory induced collapse, within a finite time, to eigenstates of the operators corresponding to total mass within 
regions of this size, and that states that are not eigenstates of these mass operators could persist only for a minuscule fraction of a second.

On such a theory, for every region of space of sufficient size, the quantum state would, almost all of the time, be an eigenstate corresponding to a definite mass within that region. Hence, by the eigenstate-eigenvalue link, there would be a matter of fact about the amount of mass within that region. Thus, a possible state of the room in which I am sitting would be one in which there was a desk-shaped region of higher mass density than its surroundings. Provided that these regions of high mass density exhibited the right sort of dynamical behaviour, there would be no problem in identifying them with desks, chairs, and laboratory equipment, and there would be no problem of ontology for collapse theories.

\subsection{Distributional ontology}

Prospects are dim for a viable collapse theory that yields precise eigenstates of total mass in any bounded region, or, indeed, precise eigenstates of any local beables. A collapse theory can, however, yield close approximations to eigenstates of appropriate local beables, such as mass smeared over sufficiently large regions.

Whether the dynamics is linear, unitary, and deterministic, as in the Schrödinger equation, or non-unitary and stochastic, initial states that are close to each other, in Hilbert space norm, evolve in approximately the same way. Thus, a state that is close to being an eigenstate of a given dynamical quantity will evolve in approximately the same way as the eigenstate that it is close to.

If we accept (as we should; see Myrvold 2018 for a fuller discussion, and also Albert 2015: 127ff.) that to be a be a physical body is nothing more and nothing less than to have a certain place in a network of dynamical and causal relations of an appropriate sort, and if we accept (as we should) that there would be no problem of interpretation of an ideal collapse theory that yielded eigenstates of the right sort of dynamical quantities, then, by the Principle of Metaphysical Continuity, we should accept that regions of space whose states are very near to eigenstates of total mass can serve as physical objects just as well as would regions of space in exact eigenstates of total mass.

Considerations such as this have led to a proposed modification of the eigenstate-eigenvalue link. 
if one wishes to attribute objective properties to individual systems one has to accept that such an attribution is legitimate even when the mean value of the projection operator on the eigenmanifold associated to the eigenvalue corresponding to the attributed property is not exactly equal to 1, but is extremely close to it (Ghirardi, Grassi, and Pearle 1990: 1298).

This modification has been dubbed, by Clifton and Monton (1999), the fuzzy link.

To say that we can ascribe a property to a system when the quantum state is such that its variance is negligibly small requires that there be a matter of fact about what the variance is. Considerations of this sort suggest a revision of the way we think about dynamical quantities, along the lines advanced by Pearle (2009). On this view, dynamical variables typically do not take on sharp values as they would classically. What they have, instead, is a distribution associated with them. These distributions, though having the formal characteristics of probability distributions, are to be thought of, not as a probability distribution over precise but unknown possessed values, but as reflecting a physical, ontological, lack of determinacy about what the value is. A limiting case would be the classical case, in which the distribution is a delta function.

On this view, the value of every dynamical variable is distributional. A collapse theory will tend to narrow the spread of the distributions of some of these quantities. When the distribution is sufficiently narrow, things will be almost exactly as if the quantity has a precise value, and, under such circumstances, we can treat the variable as if it does possess a precise value. In seeking objects that behave like our familiar macroscopic objects, it is to those variables that we should direct our attention. But the spread-out distributions of the other variables are no less part of physical reality.

\subsection{Primitive ontology as an alternative?}

Given a family of operators $\hat{M}(\boldsymbol{x})$, corresponding to a smeared mass-density centred at the point $\boldsymbol{x}$, for any quantum state $\psi$ one can define a function $m(\boldsymbol{x})$, whose value at the point $\boldsymbol{x}$ is equal to the expectation value of $\hat{M}(\boldsymbol{x})$ in state $\psi$. When Ghirardi, Grassi, and Benatti (1995) introduced the smeared mass-density as a basis for the ontology of collapse theories, their proposal was an application of the fuzzy eigenstate-eigenvalue link. They argued that the quantity $m(\boldsymbol{x})$ behaves like a mass density when - and only when - the variance of $\hat{M}(\boldsymbol{x})$ is sufficiently small as to be negligible, in which case the mass density is said to be objective. When this condition is not 
satisfied, the quantity $m(\boldsymbol{x})$, though well-defined, cannot be interpreted as a mass density, as other systems do not behave as if a quantity of mass corresponding to $m(\boldsymbol{x})$ is present. In some later works (Ghirardi and Grassi 1996; Ghirardi 1997a, 1997b) the mass density is said to be "accessible" if its variance is sufficiently small (this shift is attributed by Ghirardi and Grassi 1996, fn. 5 , to a conversation with S. Goldstein).

There is, at least apparently, a rival interpretation of $m(\boldsymbol{x})$. On this view, introduced by Goldstein (1998) and discussed extensively by Allori et al. (2008), a mass density equal at every point to the expectation value of $\hat{M}(\boldsymbol{x})$ is posited as additional, primitive ontology, over and above the quantum state.

The quantities $m(\boldsymbol{x})$ are well-defined for any quantum state. However, since, in situations in which the objectivity, or accessibility, condition is not satisfied - that is, situations in which the variance of $\hat{M}(\boldsymbol{x})$ is not small enough to be neglected-other objects do not respond as if a mass density equal to $m(\boldsymbol{x})$ is present, $m(\boldsymbol{x})$ acts like a mass density only when the accessibility condition is satisfied. Something that doesn't act like a mass density isn't a mass density. Thus, on the supposed rival interpretation, despite what is said, a mass density is present only when there is a mass density present on the original, quantum state monist proposal, that is, when the accessibility condition is satisfied. The proposal to take $m(\boldsymbol{x})$ as additional, primitive ontology does not present a genuine alternative to the original proposal of Ghirardi, Grassi, and Benatti (1995).

\section{A schema for relativistic collapse theories}

\subsection{Relativistic spacetimes}

We assume a spacetime equipped with a causal order, that is, a relation $\ll$ of causal precedence, assumed to be transitive and antisymmetric (that is, if $p$ causally precedes $q$, then $q$ does not causally precede $p$ ). Two spacetime points are said to be causally unconnected if they stand in no causal order, that is, if neither $p \ll q$ nor $q \ll p$ obtains. Because no point is in the causal past of itself, the relation of being causally unconnected is reflexive. That it is symmetric follows straightforwardly from its definition. Two distinct points that are causally unconnected are said to be spacelike separated.

In Galilean spacetime, the relation of being causally unconnected is transitive, and, therefore, is an equivalence relation, and the spacetime can be portioned into equivalence classes of 
simultaneity. In Minkowski spacetime, on the other hand, for any two points $p, q$ that are spacelike separated from each other, there are other points $r$ that are spacelike separated from $p$ but not from $q$. Define a relativistic spacetime as one in which, for any spacelike separated $p, q$, there exists a point $r$ that is spacelike separated from $p$, such that $r \ll q$.

A causal curve is a curve such that, for any pair of distinct points $p, q$, either $p \ll q$ or $q \ll p$ . A Cauchy surface is a set of spacetime points that is intersected exactly once by every inextendible causal curve. A spacetime that contains Cauchy surfaces is said to be globally hyperbolic.

We will, in what follows, assume a globally hyperbolic relativistic spacetime. We can define the relation $\leq$ between Cauchy surfaces: If $\sigma, \sigma^{\prime}$ are two Cauchy surfaces, then $\sigma \leq \sigma^{\prime}$ when no part of one if no part of $\sigma^{\prime}$ is in the causal past of $\sigma$. This relation is reflexive and transitive, and hence is a partial order on Cauchy surfaces.

\subsection{Collapse theories in relativistic spacetime}

A collapse theory modifies the deterministic, unitary evolution so as to produce something like the textbook collapse. Gisin (1989) has demonstrated, on the assumption that the evolution is Markovian (meaning that future states depend only on the present state, and not on any details about the past that aren't reflected in the present), that any deterministic, nonlinear dynamics for quantum states that does not respect a certain linearity condition permits signalling - if two spatially separated systems are in an entangled state, a choice of experiment on one can influence probabilities of outcomes of experiments performed on the other.

The relevant condition is the following.

Linearity. Let $T$ be a dynamical map on the set of pure states of a system. Let $\left\{\psi_{i}, i=1, \ldots, n\right\}$ and $\left\{\varphi_{j}, j=1, \ldots, m\right\}$ be sets of pure states such that, for some nonnegative weights $\left\{x_{\mathrm{i}}\right\},\left\{y_{j}\right\}$,

$$
\sum_{i=1}^{n} x_{i} \psi_{i}=\sum_{j=1}^{m} y_{j} \varphi_{j}
$$

Then

$$
\sum_{i=1}^{n} x_{i} T\left(\psi_{i}\right)=\sum_{j=1}^{m} y_{j} T\left(\varphi_{j}\right)
$$


As Kent (2005) has argued, though violations of linearity permit signalling, this need not be superluminal signalling. Nonetheless, ordinary quantum mechanics does not allow operations on one system to be used for signalling to another system, unless there is an interaction term between the two systems in the Hamiltonian, and we will assume that the no-signalling condition holds, and hence that the evolution is linear. This means that (unsurprisingly) a theory that produces collapse must be a theory with indeterministic dynamics.

It is convenient to work within what may be called the stochastic Tomonaga-Schwinger picture. The usual Tomonaga-Schwinger picture (see Schweber 1961: 419-422, for an introduction) is an extension of the interaction picture to a relativistic spacetime. One divides the Lagrangian density into two parts (typically regarded as the free Lagrangian density and the interaction Lagrangian),

$$
\mathcal{L}(x)=\mathcal{L}_{0}(x)+\mathcal{L}_{1}(x) .
$$

The operators representing observables are Heisenberg-picture operators for the free theory. We utilize, however, evolving state vectors; with each Cauchy surface $\sigma$ is associated a state vector $|\psi(\sigma)\rangle$. Evolution from a surface $\sigma$ to another, $\sigma^{\prime}$, differing by a small deformation $\delta \sigma$ about a point $x$, satisfies the Tomonaga-Schwinger equation:

$$
i \hbar c \frac{\delta|\psi(\sigma)\rangle}{\delta \sigma}=H_{1}(x)|\psi(\sigma)\rangle .
$$

Integration of this equation yields, for any Cauchy surfaces $\sigma$ and $\sigma^{\prime}$, a unitary mapping from $|\psi(\sigma)\rangle$ to $\left|\psi\left(\sigma^{\prime}\right)\right\rangle$.

We wish to modify this equation so as to produce collapse. On the stochastic TomonagaSchwinger picture, we work with Heisenberg-picture operators that are solutions to the standard field-theoretic equations, for free or interacting fields. The difference between states on different Cauchy surfaces is due to the stochastic modifications to the usual evolution. We will assume that the new dynamics is Markovian: that is, that, if $\sigma \leq \sigma^{\prime}$, the set of possible states on $\sigma^{\prime}$, and their respective probabilities, are determined by $|\psi(\sigma)\rangle$, and not by other facts about the history leading up to that state.

Given two Cauchy surfaces, $\sigma, \sigma^{\prime}$, with $\sigma \leq \sigma^{\prime}$, and a state vector $|\psi(\sigma)\rangle$, there will be some state vector $\left|\psi\left(\sigma^{\prime}\right)\right\rangle$, but what this vector will be is not determined by $|\psi(\sigma)\rangle$ and the dynamics. Instead, there will be some set of alternatives $\left\{\left|\psi_{\gamma}\left(\sigma^{\prime}\right)\right\rangle\right\}$, which we take to be indexed by a 
parameter $\gamma$ that takes on values in a set $\Gamma$. We expect our theories to specify, given two surfaces $\sigma, \sigma^{\prime}$, with $\sigma \leq \sigma^{\prime}$, and the state vector $|\psi(\sigma)\rangle$, the set of alternatives $\left\{\left|\psi_{\gamma}\left(\sigma^{\prime}\right)\right\rangle, \gamma \in \Gamma\right\}$, and also a probability distribution over the possible values of $\gamma$. Suppose that, with respect to some background measure $\mu$, this probability distribution is represented by a density function $p(\gamma)$.

With this apparatus in place, we can define a mixed state, $\bar{\rho}\left(\sigma^{\prime} ; \sigma\right)$ as the weighted average over the possibilities for the state on $\sigma^{\prime}$, given $|\psi(\sigma)\rangle$.

$$
\bar{\rho}\left(\sigma^{\prime} ; \sigma\right)=\int_{\gamma} p(\gamma)\left|\psi_{\gamma}\left(\sigma^{\prime}\right)\right\rangle\left\langle\psi_{\gamma}\left(\sigma^{\prime}\right)\right| d \mu
$$

This would be the state used by someone who knows the state on $\sigma$ and the possible state transitions from $|\psi(\sigma)\rangle$ to $\left|\psi\left(\sigma^{\prime}\right)\right\rangle$ and their respective probabilities, but does not know the outcome of the process that occurs between $\sigma$ and $\sigma^{\prime}$.

Gisin's proof, mentioned above, generalizes to stochastic theories. If we take $T$ to be the mapping that takes a pure state on $\sigma$ to a mixed state $\bar{\rho}\left(\sigma^{\prime} ; \sigma\right)$, no-signalling entails that this map must satisfy the linearity condition (Simon, Bužek, and Gisin 2001, Bassi and Hejazi 2015), and from this, together with the condition that, applied to subsystems in entangled states, the mapping extend to a positive map on the state space of the wider system, entails that the map from the state on $\sigma$ to the mixed state $\bar{\rho}\left(\sigma^{\prime} ; \sigma\right)$ be a completely positive map.

We will therefore take the mapping from a pure state on $\sigma$ to the mixed state $\bar{\rho}\left(\sigma^{\prime} ; \sigma\right)$ to be a nonselective completely positive map, which is a mixture of selective completely positive maps that takes us from $|\psi(\sigma)\rangle$ to $\left|\psi_{\gamma}\left(\sigma^{\prime}\right)\right\rangle$. This entails that there is a set of operators $\left\{K_{\gamma}, \gamma \in \Gamma\right\}$, which we will call evolution operators, such that, for some $\gamma$,

$$
\left|\psi\left(\sigma^{\prime}\right)\right\rangle=\left|\psi_{\gamma}\left(\sigma^{\prime}\right)\right\rangle=K_{\gamma}|\psi(\sigma)\rangle / \| K_{\gamma}|\psi(\sigma)\rangle \|
$$

with probabilities for which state is realized given by

$$
\operatorname{Pr}(\gamma \in \Delta)=\int_{\Delta} p(\gamma) d \mu
$$

The linearity condition entails that

$$
p(\gamma)=\| K_{\gamma}|\psi(\sigma)\rangle \|^{2}
$$

Any probabilities that depart from these would lead to signalling. The condition that $p$ always be normalized is the condition that 


$$
\int_{\Gamma} K_{\gamma}^{\dagger} K_{\gamma} d \mu=1
$$

The evolutions should also satisfy the semi-group property, which requires that, for Cauchy surfaces $\sigma \leq \sigma^{\prime} \leq \sigma^{\prime \prime}$, the possible evolutions from $\sigma$ to $\sigma^{\prime \prime}$ be the compositions of evolutions from $\sigma$ to $\sigma^{\prime}$ with evolutions from $\sigma^{\prime}$ to $\sigma^{\prime \prime}$.

The theory of quantum dynamical semi-groups is well-studied (see Bassi and Ghirardi 2003, or Alicki and Lendi 2007 for an introduction). Provided that the evolution satisfies an appropriate continuity condition, the mixed-state density operators on Cauchy surfaces $\sigma$ to the future of some surface $\sigma_{0}$ will satisfy a Lindblad equation. We consider the change in $\bar{\rho}\left(\sigma ; \sigma_{0}\right)$ as we pass from one surface $\sigma$ to another, $\sigma^{\prime}$, differing by a small deformation about a point $x$ on $\sigma$, with spacetime volume $\delta \sigma$. Let $H(x)$ be the Hamiltonian density, that is, the component of the energymomentum density along the normal to $\sigma$ at $x$. For a Lindblad-type evolution, there is also a countable set $\left\{L_{\alpha}(x)\right\}$ of operators, such that the change $\delta \bar{\rho}$ satisfies

$$
\frac{\delta \bar{\rho}}{\delta \sigma}=\frac{1}{i \hbar c}[H(x), \bar{\rho}]+\sum_{\alpha} L_{\alpha}(x) \bar{\rho} L_{\alpha}^{\dagger}(x)-\frac{1}{2}\left(\sum_{\alpha} L_{\alpha}^{\dagger}(x) L_{\alpha}(x) \bar{\rho}+\bar{\rho} \sum_{\alpha} L_{\alpha}^{\dagger}(x) L_{\alpha}(x)\right) .
$$

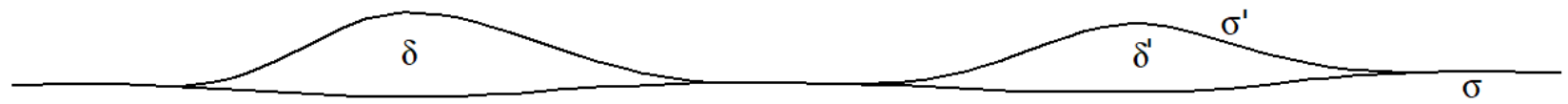

Figure 1. Two Cauchy surfaces $\sigma, \sigma^{\prime}$, with $\sigma \leq \sigma^{\prime}$, that coincide everywhere except on the boundaries of two bounded regions $\delta$ and $\delta^{\prime}$.

Consider two Cauchy surfaces $\sigma, \sigma^{\prime}$, with $\sigma \leq \sigma^{\prime}$, that coincide everywhere except on the boundaries of two bounded regions $\delta$ and $\delta^{\prime}$ (see Figure 1). The evolution from $\sigma$ to $\sigma^{\prime}$ through $\delta \cup \delta^{\prime}$ must equal the composition of the evolution through $\delta$ and the evolution through $\delta^{\prime}$, in either order. The necessary and sufficient condition for this is that evolution operators corresponding to spacelike separated regions commute.

Moreover, for computing probabilities for the results of experiments on the overlap of $\sigma$ and $\sigma^{\prime}$, it should not matter whether $|\psi(\sigma)\rangle$ or $\bar{\rho}\left(\sigma^{\prime} ; \sigma\right)$ is used. Someone located in the overlap, who knows the state on $\sigma$ and believes collapse will occur between $\sigma$ and $\sigma^{\prime}$, but does not know (because it occurs at spacelike separation) what the outcome of that collapse is, should be able to 
use $|\psi(\sigma)\rangle$ or $\bar{\rho}\left(\sigma^{\prime} ; \sigma\right)$ for computing probabilities of results of experiments that she is about to undertake, and these should yield the same probabilities for outcomes of those experiments. The necessary and sufficient condition for this is that evolution operators that implement evolution through a given spacetime region $\delta$ should commute with operators representing observables at spacelike separation from $\delta$.

These conditions give us a rather general schema for a quantum theory with stochastic dynamics on a relativistic spacetime. It includes, as a special case, deterministic, unitary evolution, in which case the set of evolution operators pertaining to any region of spacetime is a singleton set. Concrete theories will fill in the details, specifying, in particular, what the sets of evolution operators are.

\section{Beables for relativistic collapse theories}

\subsection{Intrinsic and extrinsic states of a spacetime region}

Consider a bounded spacelike region $\alpha$, that is common to Cauchy surfaces $\left\{\sigma, \sigma^{\prime}, \sigma^{\prime \prime}, \ldots\right\}$. In the stochastic Tomonaga-Schwinger picture, there will be quantum states $\rho(\sigma), \rho\left(\sigma^{\prime}\right), \rho\left(\sigma^{\prime \prime}\right), \ldots$ Each of these states yields probabilities of outcomes of experiments to the future of its Cauchy surface, conditional on events, including any collapses, to the past of the Cauchy surface. For each of these states, we can consider the reduced state that consists of the restriction of the state to observables in the forward domain of dependence of $\alpha$. Call these reduced states $\rho_{\alpha}(\sigma), \rho_{\alpha}\left(\sigma^{\prime}\right)$, etc.. If the evolution between two surfaces $\sigma$ and $\sigma^{\prime}$ is purely unitary, then $\rho_{\alpha}(\sigma)$ will coincide with $\rho_{\alpha}\left(\sigma^{\prime}\right)$. If, however, collapse occurs between $\sigma$ and $\sigma^{\prime}$, then they need not coincide.

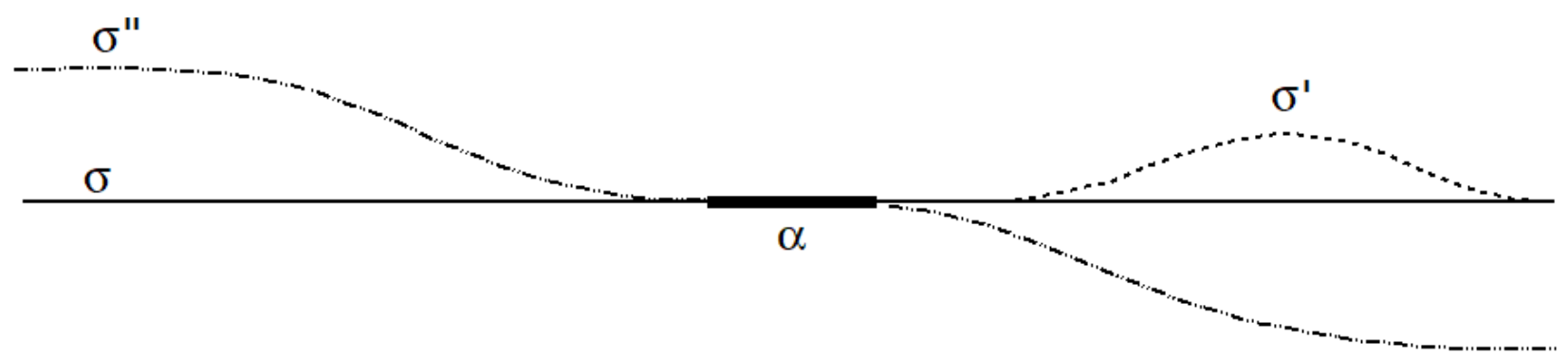

Figure 2. Three Cauchy surfaces containing a common part $\alpha$. 
Since the reduced state $\rho_{\alpha}(\sigma)$ is conditioned on any collapses to the past of $\sigma$, including any that are spacelike separated from $\alpha$, it should be clear that, though it is associated with the region $\alpha, \rho_{\alpha}(\sigma)$ cannot in general be regarded as a beable local to $\alpha$. Thus, if the reduced states $\rho_{\alpha}(\sigma)$ and $\rho_{\alpha}\left(\sigma^{\prime}\right)$ differ, they do not offer competing accounts of intrinsic properties of the region $\alpha$.

The intrinsic state of a bounded spacelike region $\alpha$ must be conditioned only on collapses to the past of $\alpha$. We can define this state by a limiting procedure. Consider a sequence of Cauchy surfaces $\left\{\sigma_{1}, \sigma_{2}, \ldots, \sigma_{n}, \ldots\right\}$, that is such that $\alpha$ is contained as a common part of all $\sigma_{n}$ and for all $n, \sigma_{n+1} \leq \sigma_{n}$, and the sequence converges on the past light cone of $\alpha$ (that is, the set of points that are to the past of all $\sigma_{n}$ is precisely the casual past of $\alpha$. Define the past light-cone state of $\alpha$ as the limit, if it exists, of $\rho_{\alpha}\left(\sigma_{n}\right)$, as $n$ increases indefinitely. Though a state derived from a Cauchy surface with events to its past that are spacelike separated from $\alpha$ cannot be regarded as the intrinsic state of $\alpha$, its past light-cone state can.

\subsection{Compatibility of extrinsic states}

Maudlin (1996: 301-302) raised the question of consistency of state assignments derived from different hypersurfaces passing through a given region. If two hypersurfaces $\sigma, \sigma^{\prime}$ having a region $\alpha$ in common, yielded reduced states that were orthogonal to each other, in the sense of yielding conflicting definite (probability equal to unity) predictions for the outcome of some experiment, this would be problematic.

The question arises: do the conditions on collapse dynamics outlined above guarantee that the differing extrinsic state assignments obtained from different Cauchy surfaces are not in outright conflict with each other? It can be shown (see Myrvold 2003: 489, 2016: 255-257) that these conditions suffice to guarantee that the states states $\rho_{\alpha}(\sigma)$ and $\rho_{\alpha}\left(\sigma^{\prime}\right)$ are not orthogonal.

In fact, a stronger sense of compatibility obtains. The question of the compatibility of reduced states derived from states on different Cauchy surfaces is essentially the same as that addressed by Brun, Finkelstein, and Mermin (2002). They demonstrate that state assignments that can represent information about a system available to different observers are compatible, in the sense that they have overlapping support.

The context in which Brun et al. work is that of finite-dimensional Hilbert spaces. However, essentially the same conclusion holds in a setting appropriate to quantum field theory. In this 
context we cannot assume a finite-dimensional Hilbert space, nor can we assume that the mixed state of a bounded region $\alpha$ obtained from a pure state on a Cauchy surface containing $\alpha$ admits of a decomposition into pure states. We must take care to formulate the condition in a manner that is independent of assumptions such as these.

We assume a von Neumann algebra $R(\alpha)$, whose self-adjoint elements represent the bounded observables pertaining to the forward domain of dependence of $\alpha$. Let $\rho$ be a normal state of $R(\alpha)$ (that is, a completely additive state). We define the support projection for $\rho$ as the orthogonal complement of the union of all projections in $R(\alpha)$ to which $\rho$ assigns expectation value zero.

With these definitions in hand, it can be shown that, given a set $\left\{\sigma, \sigma^{\prime}, \sigma^{\prime \prime}, \ldots\right\}$ of Cauchy surfaces containing $\alpha$, then on the assumption that there is a Cauchy surface containing $\alpha$ that is nowhere to the past of any of them (which, in particular, will always be the case for any finite set of Cauchy surfaces), the corresponding set of states $\left\{\rho_{\alpha}(\sigma), \rho_{\alpha}\left(\sigma^{\prime}\right), \rho_{\alpha}\left(\sigma^{\prime \prime}\right), \ldots\right\}$ have nonzero overlapping support. See Appendix for details.

\subsection{Local beables for collapse theories}

Suppose that we have a collapse theory that yields near-eigenstates of an appropriate dynamical quantity. For example: the natural extension to the relativistic context of a mass-density would be the components of the stress-energy tensor. Assume that we have an appropriate relativistically invariant smearing function (see Bedingham 2011a,b), and formulate smeared operators $\hat{T}_{\mu v}(x)$, representing a smeared stress-energy density centred at the point $x$. For any state $\rho$, we can define

$$
\mathcal{T}_{\mu \nu}(x)=\left\langle\hat{T}_{\mu \nu}(x)\right\rangle_{\rho}
$$

The 00-component of this is the relativistic analogue of the mass density that has been proposed as an appropriate ontology for nonrelativistic collapse theories.

For a bounded region $\alpha$ contained in distinct Cauchy surfaces $\sigma$ and $\sigma^{\prime}$, the reduced states $\rho_{\alpha}(\sigma)$ and $\rho_{\alpha}\left(\sigma^{\prime}\right)$ may yield differing values for $\mathcal{T}_{\mu v}(x)$, with $x$ within $\alpha$. But, obviously, these do not yield rival accounts of local beables within $\alpha$; as they are defined via the extrinsic states $\rho_{\alpha}(\sigma)$ and $\rho_{\alpha}\left(\sigma^{\prime}\right)$, which are not themselves local beables. 
Where, then, may we find local beables for a relativistic collapse theory? There are, in the literature, two proposals for extending the fuzzy link to a relativistic context. One is what might be called the agreement criterion, formulated by Ghirardi, Grassi, and Pearle (1991):

We think that the appropriate attitude is the following: when considering a local observable $A$ with its associated support we say that an individual system has the objective property $a$ ( $a$ being an eigenvalue of $A$ ), only when the mean value of $P a$ is extremely close to one, when evaluated on all spacelike hypersurfaces containing the support of $A$. (1991: 1310).

This means that, for $x$ within $\alpha, \mathcal{T}_{\mu v}(x)$ will be regarded as representing an objective property if and only if the accessibility criterion is satisfied in $\rho_{\alpha}(\sigma)$ for every Cauchy surface containing $\alpha$

The other criterion is the past light cone criterion, formulated by Ghirardi and Grassi (1994: 419; see also Ghirardi 1996: 336, 1999: 139, 2000: 1364). On this criterion, a system is said to possess the property $A=a$ when the expectation value of $P a$ is extremely close to one, evaluated on the past light-cone state.

If the criterion for property attribution were an exact eigenstate-eigenvalue link - that is, if we were ascribing a property $A=a$ only when the expectation value of $P a$ is exactly equal to onethen the two would be equivalent. The past light-cone state of a region $\alpha$ is an eigenstate of an observable $A$ pertaining to $\alpha$ with eigenvalue $a$ if and only if the state on every Cauchy surface containing $\alpha$ is. On the fuzzy link, the agreement criterion entails the past light-cone criterion, but the past light-cone criterion does not guarantee satisfaction of the agreement criterion; it only entails that the agreement criterion will hold with high probability.

If the property attribution criterion is meant to supply local beables, then it is clear that what is wanted is the past light-cone criterion and not the agreement criterion. The agreement criterion makes reference to events at spacelike separation from the region in question. Moreover, as Ghirardi, Grassi, Butterfield, and Fleming (1993: 358) have shown, a choice regarding experiments performed at spacelike separation from $\alpha$ can affect the probability that the agreement criterion is satisfied. Consider a case of two spin- $1 / 2$ particles, located in world-tubes $A$ and $B$. We take initial conditions on a Cauchy surface $\sigma_{0}$, and suppose that the particle in $A$ is undisturbed in the interval between $\sigma_{0}$ and some later Cauchy surface $\sigma_{1}$. Let $\alpha$ be a spacelike slice of $A$ between $\sigma_{0}$ and $\sigma_{1}$. 
Let $\beta_{0}, \beta_{1}$ be the intersections of $B$ with $\sigma_{0}$ and $\sigma_{1}$, respectively. We will take $\sigma_{0}$ and $\sigma_{1}$ such that $\beta_{0}$ and $\beta_{1}$ are to the past, and future, respectively, of $\alpha$ (see Figure 3).

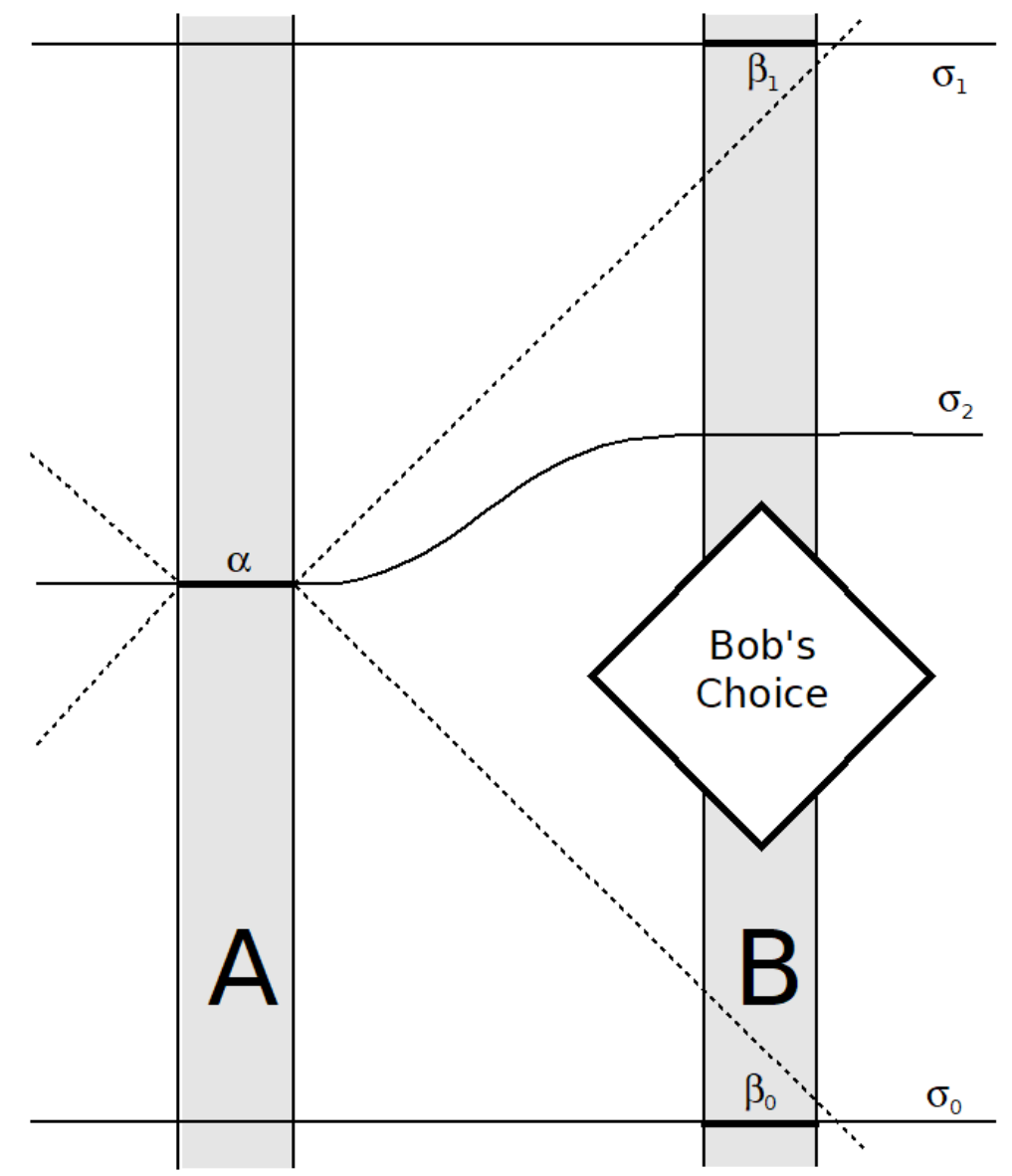

Figure 3. Set-up for the example described in the text.

Suppose that the state of the pair of particles on $\sigma_{0}$ is

$$
\left|\psi\left(\sigma_{0}\right)\right\rangle=\sqrt{1-\varepsilon}|+\rangle_{A}|+\rangle_{B}+\sqrt{\varepsilon}|-\rangle_{A}|-\rangle_{B}
$$

where $|+\rangle$ and $|-\rangle$ are spin eigenstates in some designated direction (say, the $z$-direction), and $\varepsilon$ is an extremely small number, small enough that the state $\left|\psi\left(\sigma_{0}\right)\right\rangle$ is sufficiently close to an eigenstate of spin for the particle in $A$ that the accessibility criterion is satisfied. Thus, on the past light-cone criterion, we ascribe + spin in the $z$-direction to the particle in $A$ as a possessed property. 
Suppose that Bob, located near $B$, has a choice of whether to perform a spin experiment on $B$. Suppose that, if he doesn't, the pair of spin- $-1 / 2$ systems is effectively isolated from outside interference, and that in that case our collapse theory assigns, for some $\delta$ smaller than $\varepsilon$, probability $1-\delta$ that the state will remain undisturbed in the interval between $\sigma_{0}$ and $\sigma_{1}$, in which case the agreement criterion for property attribution is satisfied. If Bob chooses to do a spin experiment on the particle in $B$, there is probability $\varepsilon$ that he will obtain the result $|-\rangle$. If he does, then the state of the combined system on a Cauchy surface $\sigma_{2}$ that includes $\alpha$ and runs to the future of Bob's experiment will not be a state in which the particle in $A$ is close to a + eigenstate for spin- $z$; on the contrary, it will close to a - eigenstate for spin- $z$. In such an eventuality, the agreement criterion for ascribing 'spin- $z=+$ ' to the particle in $A$ is not satisfied.

Now, if the threshold for satisfaction of the accessibility criterion is stringent enough - say, $10^{-40}$, as suggested by Pearle (1997) — then the probability of disagreement between the past lightcone criterion and the agreement criterion is sufficiently low as to be negligible, whether or not Bob chooses to do an experiment. However, it is still true that the value of this negligibly low probability depends on Bob's choice regarding his experiment, and hence, if we were to apply the agreement criterion for outcome attribution, this would require acceptance that the theory exhibits parameter dependence, albeit a very weak parameter dependence (see Ghirardi, Grassi, Butterfield, and Fleming 1993 for discussion). If, however, we adopt the past light-cone criterion, then (as noted already by Ghirardi and Grassi 1994), there is no parameter dependence at all, not even very weak dependence. The conclusion to be drawn is that local beables for a relativistic collapse theory are to be identified according to the past light-cone criterion.

As mentioned, Ghirardi, Grassi, and Benatti (1995) have argued that a theory on which, at the macroscopic scales, a smeared mass density is almost always near-definite yields an adequate picture of the world. Combined with the past light-cone criterion, this gives a past light-cone matter density ontology, discussed by Tumulka (2007) and, in more detail, by Bedingham et al. (2014).

\section{Conclusion}

There is a sensible ontology for collapse theories in a relativistic context. Moreover, considerations of what it takes for a theory to represent a world that contains, among other things, objects like our experimental apparatus, to be thought of a local beables, determine the form that this ontology 
takes. It is one on which all dynamical quantities are distributional in character. In spite of this distributional character, dynamical quantities may hace effectively precise values (in the sense that they behave, to a high degree of approximation, as if they have precise values); it is the goal of a collapse theory to ensure that the properties of macroscopic objects almost always have this character. Beables local to a bounded spacetime region are to be evaluated via the past light cone state of that region.

\section{Appendix}

We consider a finite set of Cauchy surfaces $\left\{\sigma_{1}, \sigma_{2}, \ldots, \sigma_{n}\right\}$, all containing an open subset $\alpha$. Our goal is to show that, given the conditions on a relativistic collapse theory, the states $\rho_{\alpha}\left(\sigma_{1}\right), \rho_{\alpha}\left(\sigma_{2}\right), \ldots, \rho_{\alpha}\left(\sigma_{n}\right)$ have common support.

We assume a Hilbert space that contains vectors $\left|\psi\left(\sigma_{1}\right)\right\rangle,\left|\psi\left(\sigma_{2}\right)\right\rangle, \ldots,\left|\psi\left(\sigma_{n}\right)\right\rangle$. We assume also that, if $\sigma \leq \sigma^{\prime}$, there exists $K_{\gamma}$ such that

$$
\left|\psi\left(\sigma^{\prime}\right)\right\rangle=K_{\gamma}|\psi(\sigma)\rangle / \| K_{\gamma}|\psi(\sigma)\rangle \|
$$

If $\sigma \leq \sigma^{\prime}$, and $\alpha$ is in the overlap of $\sigma$ and $\sigma^{\prime}$, then the region between $\sigma$ and $\sigma^{\prime}$ is spacelike separated from $\alpha$. Therefore, $K_{\gamma}$ commutes with all self-adjoint elements of $R(\alpha)$.

The restrictions of the states on the Cauchy surfaces $\sigma_{1}, \sigma_{2}, \ldots, \sigma_{n}$ are states (which will typically be mixed states) of $R(\alpha), \rho_{\alpha}\left(\sigma_{1}\right), \rho_{\alpha}\left(\sigma_{2}\right), \ldots, \rho_{\alpha}\left(\sigma_{n}\right)$. We do not assume that these are represented by density operators in $R(\alpha)$, or that they are mixtures of pure states of $R(\alpha)$, as this is not needed in what follows.

As mentioned in the main text, the projector onto the null space of any state $\rho$ of $R(\alpha)$ is the union of all projections $P$ that have zero expectation value in $\rho$, and the support projection of $\rho$ is the orthogonal complement of the projector onto the null space. We will call the null space and the support of $\rho, \operatorname{Null}[\rho]$ and $\operatorname{Supp}[\rho]$.

Lemma 1. Let $\sigma$ and $\sigma^{\prime}$ be Cauchy surfaces containing a common open subset $\alpha$. If $\sigma \leq \sigma^{\prime}$, then, for any positive operator $E \in R(\alpha)$, if $\langle\psi(\sigma)|E| \psi(\sigma)\rangle=0$, then $\left\langle\psi\left(\sigma^{\prime}\right)|E| \psi\left(\sigma^{\prime}\right)\right\rangle=0$.

Proof. Any positive operator $E$ has a square root $E^{1 / 2}$. Suppose that 


$$
\langle\psi(\sigma)|E| \psi(\sigma)\rangle=0
$$

Therefore, since

$$
\langle\psi(\sigma)|E| \psi(\sigma)\rangle=\| E^{1 / 2}|\psi(\sigma)\rangle \|^{2}
$$

then

$$
E^{1 / 2}|\psi(\sigma)\rangle=0
$$

For some $K_{\gamma}$ that commutes with all self-adjoint $E \in R(\alpha)$,

$$
\left|\psi\left(\sigma^{\prime}\right)\right\rangle=K_{\gamma}|\psi(\sigma)\rangle / \| K_{\gamma}|\psi(\sigma)\rangle \|,
$$

Therefore,

$$
E^{1 / 2}\left|\psi\left(\sigma^{\prime}\right)\right\rangle=E^{1 / 2} K_{\gamma}|\psi(\sigma)\rangle / \| K_{\gamma}|\psi(\sigma)\rangle \|=K_{\gamma} E^{1 / 2}|\psi(\sigma)\rangle / \| K_{\gamma}|\psi(\sigma)\rangle \|=0
$$

and so

$$
\left\langle\psi\left(\sigma^{\prime}\right)|E| \psi\left(\sigma^{\prime}\right)\right\rangle=0
$$

Lemma 1 gives us a relation between the supports of $\rho_{\alpha}(\sigma)$ and $\rho_{\alpha}\left(\sigma^{\prime}\right)$ when $\sigma \leq \sigma^{\prime}$.

Proposition 1. Let $\sigma$ and $\sigma^{\prime}$ be Cauchy surfaces containing a common open subset $\alpha$. If $\sigma \leq \sigma^{\prime}$, then $\operatorname{Null}\left[\rho_{\alpha}(\sigma)\right] \subseteq N u l l\left[\rho_{\alpha}\left(\sigma^{\prime}\right)\right] ;$ equivalently, $\operatorname{Supp}\left[\rho_{\alpha}\left(\sigma^{\prime}\right)\right] \subseteq \operatorname{Supp}\left[\rho_{\alpha}(\sigma)\right]$.

Proof. This is immediate from Lemma 1.

From this follows the result concerning overlapping support of $\left\{\rho_{\alpha}\left(\sigma_{1}\right), \rho_{\alpha}\left(\sigma_{2}\right), \ldots, \rho_{\alpha}\left(\sigma_{n}\right)\right\}$.

Proposition 2. Let $\sigma_{1}, \sigma_{2}, \ldots, \sigma_{n}$ be Cauchy surfaces sharing a common open subset $\alpha$. Then the supports of $\rho_{\alpha}\left(\sigma_{1}\right), \rho_{\alpha}\left(\sigma_{2}\right), \ldots, \rho_{\alpha}\left(\sigma_{n}\right)$ have nonzero intersection.

Proof. We can construct a Cauchy surface $\sigma_{+}$that contains $\alpha$ and is such that $\sigma_{i} \leq \sigma_{+}$for each $i$, by taking the least upper bound of the set $\left\{\sigma_{1}, \sigma_{2}, \ldots, \sigma_{n}\right\}$ under the ordering $\leq$. Consider, now, the state $\rho_{\alpha}\left(\sigma_{+}\right)$. By Proposition 1, Supp $\left[\rho_{\alpha}\left(\sigma_{+}\right)\right] \subseteq \operatorname{Supp}\left[\rho_{\alpha}\left(\sigma_{i}\right)\right]$ for all $i$, and hence the support of $\sigma_{+}$lies within the intersection of the supports of $\rho_{\alpha}\left(\sigma_{1}\right), \rho_{\alpha}\left(\sigma_{2}\right), \ldots, \rho_{\alpha}\left(\sigma_{n}\right)$. 
Remark I. The restriction to a finite set is unnecessary; the result holds for an infinite set of Cauchy surfaces provided that there is a Cauchy surface that is the upper bound of all of them. Furthermore, if there is a future light-cone state that is the limit of an increasing (in the ordering $\leq$ ) set of Cauchy surfaces that converges on the future light cone of $\alpha$, then the support of the future light cone states is the intersection of the supports of all $\rho_{\alpha}(\sigma)$ for all $\sigma$ containing $\alpha$.

Remark II. A quantum field is an assignment of a "field operator" $\hat{\varphi}(x)$ to each point of spacetime. In standard quantum field theories on Minkowksi spacetime, it is assumed that there is a unitary representation of the group of spacetime translations, with infinitesimal generators $P^{\mu}$ that satisfy the spectrum condition:

For any future-directed timelike vector $a$, the spectrum of $\mathrm{Pa}$ is in $\mathbb{R}^{+}$

This ensures positivity of the energy, with respect to any reference frame.

We assume a unique vacuum state that is invariant under all spacetime symmetries. Define the standard Hilbert space of the theory as the closure in norm of the set of all vectors that can be obtained by operating on the vacuum state with operators constructed from standard fields. It follows from the Reeh-Schlieder theorem that, for any state $\rho$ that is analytic in energy, for any $\alpha$ that is such that the set of points spacelike separated from $\alpha$ contains an open set, the null space of $\rho$ is empty. If $\rho_{\alpha}\left(\sigma_{1}\right), \rho_{\alpha}\left(\sigma_{2}\right), \ldots, \rho_{\alpha}\left(\sigma_{n}\right)$ are all states in the standard Hilbert space of the theory that are analytic in the energy, each of their null spaces consists solely of the zero vector, and hence Proposition 2 holds trivially.

The proposition is less trivial for theories that introduce nonstandard fields, and whose states go beyond the standard Hilbert space, as do the relativistic versions of CSL due to Bedingham $(2011 a, b)$ and Pearle (2015). It can be shown, for any indeterministic theory formulated within the framework sketched in Section 3 that is set in Minkowski spacetime, it is necessary to go beyond the standard Hilbert space (see Myrvold 2017).

\section{Acknowledgments}

Many thanks to the organizers of the workshop, Identity, indistinguishability and non-locality in quantum physics, Buenos Aires, June 2017, and to the participants in that workshop, for their helpful comments. Thanks also to Philip Pearle for comments and advice. 
I am grateful to Graham and Gale Wright, who generously sponsor the Graham and Gale Wright Distinguished Scholar Award at the University of Western Ontario, for financial support of this work.

\section{References}

Albert, D. Z. (1996). "Elementary quantum metaphysics," pp. 277-284 in J. T. Cushing, A. Fine, and S. Goldstein (eds.), Bohmian Mechanics and Quantum Mechanics: An Appraisal. Dordrecht: Kluwer Academic Publishers.

Albert, D. Z. (2015). After Physics. Cambridge, MA: Harvard University Press.

Albert, D. Z. and Loewer, B. (1991). "Wanted dead or alive: Two attempts to solve Schrödinger's paradox," pp. 278-285 in A. Fine, M. Forbes, and L. Wessels (eds.), PSA 1990: Proceedings of the 1990 Biennial Meeting of the Philosophy of Science Association, Volume One: Contributed Papers. East Lansing, MI: Philosophy of Science Association.

Alicki, R., and Lendi, K. (2007). Quantum Dynamical Semigroups and Applications, 2nd ed. Berlin: Springer.

Allori, V., Goldstein, S., Tumulka, R., and Zanghí, N. (2008). "On the common structure of Bohmian mechanics and the Ghirardi-Rimini-Weber theory," The British Journal for the Philosophy of Science 59: 353-389.

Bassi, A. and Ghirardi, G. C. (2003). "Dynamical reduction models," Physics Reports, 379: 257426.

Bassi, A. and Hejazi, K. (2015). "No-faster-than-light-signaling implies linear evolution. A rederivation.” European Journal of Physics, 36: 055027.

Bassi, A., Lochan, K., Satin, S., Singh, T., and Ulbricht, H. (2013). "Models of wave-function collapse, underlying theories, and experimental tests," Reviews of Modern Physics, 85: 471527.

Bedingham, D. (2011a). "Relativistic state reduction model." Journal of Physics: Conference Series, 306: 012034. 
Bedingham, D. (2011b). "Relativistic state reduction dynamics," Foundations of Physics, 41: 686704.

Bedingham, D., Dürr, D., Ghirardi, G. C., Goldstein, S., Tumulka, R., and Zanghi, N. (2014). "Matter density and relativistic models of wave function collapse," Journal of Statistical Physics, 154: 623-631.

Brun, T., Finkelstein, J., and Mermin, N. D. (2002). "How much state assignments can differ," Physical Review A 65: 032315.

Clifton, R. and Monton, B. (1999). "Losing your marbles in wavefunction collapse theories," The British Journal for the Philosophy of Science, 50: 697-717.

Diósi, L. (1989). "Models for universal reduction of macroscopic quantum fluctuations," Physical Review A 40: 1165-1174.

Dove, C. (1996). Explicit Wavefunction Collapse and Quantum Measurement. Ph.D. thesis. Durham: Department of Mathematical Sciences, University of Durham.

Dove, C. and Squires, E. J. (1996). “A local model of explicit wavefunction collapse,” arXiv:quant$\mathrm{ph} / 9605047$.

Ghirardi, G. C. (1996). "Properties and events in a relativistic context: Revisiting the dynamical reduction program," Foundations of Physics Letters, 9, 313-355.

Ghirardi, G. C. (1997a). "Quantum dynamical reduction and reality: Replacing probability densities with densities in real space," Erkenntnis, 45: 349-65.

Ghirardi, G. C. (1997b). "Macroscopic reality and the dynamical reduction program,” pp. 221-240 in M. L. D. Chiara, K. Doets, D. Mundici, and J. V. Benthem (eds.), Structures and Norms in Science: Volume Two of the Tenth International Congress of Logic, Methodology, and Philosophy of Science. Dordrecht: Kluwer Academic Publishers.

Ghirardi, G. C. (1999). "Some lessons from relativistic reduction models," pp. 117-52 in H.-P. Breuer and F. Petruccione (eds.), Open Systems and Measurement in Relativistic Quantum Theory: Proceedings of the Workshop Held at the Istituto Italiano per gli Studi Filosofici, Naples, April 3, 1998 (Berlin: Springer). 
Ghirardi, G. C. (2000). "Local Measurements of Nonlocal Observables and the Relativistic Reduction Process," Foundations of Physics 30: 1337-1385.

Ghirardi, G. C. (2016). "Collapse Theories,” The Stanford Encyclopedia of Philosophy (Spring 2016 Edition), E. N. Zalta (ed.), URL = <https://plato.stanford.edu/archives/spr2016/entries/qm-collapse/>.

Ghirardi, G. C. and Grassi, R. (1994). "Outcome predictions and property attribution: The EPR argument reconsidered," Studies in History and Philosophy of Science, 25: 397-423.

Ghirardi, G. C. and Grassi, R. (1996). "Bohm's theory versus dynamical reduction,” pp. 353-377 in J. T. Cushing, A. Fine, and S. Goldstein (eds.), Bohmian Mechancs and Quantum Theory: An Appraisal. Dordrecht: Kluwer Academic Publishers.

Ghirardi, G.C., Grassi, R., and Benatti, F. (1995). "Describing the macroscopic world: Closing the circle within the dynamical reduction program," Foundations of Physics, 25: 5-38.

Ghirardi, G. C., Grassi, R., and Pearle, P. (1990). "Relativistic dynamical reduction models: General framework and examples," Foundations of Physics, 20: 1271-1316.

Ghirardi, G. C., Grassi, R., Butterfield, J., and Fleming, G. N. (1993). "Parameter dependence and outcome dependence in dynamical models for state vector reduction," Foundations of Physics 23: 341-64.

Ghirardi, G. C., Grassi, R., and Pearle, P. (1991). "Relativistic dynamical reduction models and nonlocality," pp. 109-123 in P. Lahti and P. Mittelstaedt (eds.), Symposium on the Foundations of Modern Physics 1990. Singapore: World Scientific.

Ghirardi, G. C., Pearle, P., and Rimini, A. (1990). "Markov processes in Hilbert space and continuous spontaneous localization of systems of identical particles," Physical Review A, 42: 78-89.

Ghirardi, G., Rimini, A., and Weber, T. (1986). "Unified dynamics for microscopic and macroscopic systems," Physical Review D, 34: 470-491.

Gisin, N. (1989). "Stochastic quantum dynamics and relativity," Helvetica Physica Acta, 62: 363371.

Goldstein, S. (1998). "Quantum theory without observers—Part two," Physics Today 51, 38-42. 
Kent, A. (2005). "Nonlinearity without superluminality," Physical Review A, 72: \#012108.

Maudlin, T. (1996). "Space-time in the quantum world," pp. 285-307 in J. T. Cushing, A. Fine, and S. Goldstein (eds.), Bohmian Mechancs and Quantum Theory: An Appraisal. Dordrecht: Kluwer Academic Publishers.

Maudlin, T. (2007). "Completeness, supervenience and ontology," Journal of Physics A: Mathematical and Theoretical, 40: 3151-3171.

Myrvold, W. C. (2003). "Relativistic quantum becoming," The British Journal for the Philosophy of Science, 53: 475-500.

Myrvold, W. C. (2011). "Nonseparability, classical and quantum," The British Journal for the Philosophy of Science, 62: 417-432.

Myrvold, W. C. (2016). "Lessons of Bell's theorem: nonlocality, yes; action at a distance, not necessarily," pp. 238-260 in S. Gao and M. Bell (eds.), Quantum Nonlocality and Reality: 50 Years of Bell's Theorem. Cambridge: Cambridge University Press.

Myrvold, W. C. (2017). "Relativistic Markovian dynamical collapse theories must employ nonstandard degrees of freedom," Physical Review A, 96: 062116.

Myrvold, W. C. (2018). “Ontology for collapse theories," pp. 99-126 in S. Gao (ed.), Collapse of the Wave Function: Models, Ontology, Origin, and Implications. Cambridge: Cambridge University Press.

Ney, A. and Albert, D. Z. (eds.) (2013). The Wave Function: Essays on the Metaphysics of Quantum Mechanics. Oxford: Oxford University Press.

Pearle, P. (1989). "Combining stochastic dynamical state-vector reduction with spontaneous localization," Physical Review A, 39: 2277-2289.

Pearle, P. (1997). “Tales and tails and stuff and nonsense," pp. 143-156 in R. S. Cohen, M. Horne, and J. Stachel (Eds.), Experimental Metaphysics: Quantum Mechanical Studies for Abner Shimony, Volume One (Dordrecht: Kluwer Academic Publishers).

Pearle, P. (2009). "How stands collapse II," pp. 257-292 in W. C. Myrvold and J. Christian (eds.), Quantum Reality, Relativistic Causality, and Closing the Epistemic Circle: Essays in Honour of Abner Shimony. Berlin: Springer. 
Pearle, P. (2015). "Relativistic dynamical collapse model," Physical Review D, 91: 105012.

Schweber, S. (1961). An Introduction to Relativistic Quantum Field Theory. New York: Harper \& Row.

Shimony, A. (1991). "Desiderata for a modified quantum mechanics," pp. $49-59$ in A. Fine, M. Forbes, and L. Wessels (eds.), PSA 1990: Proceedings of the 1990 Biennial Meeting of the Philosophy of Science Association, Volume Two: Symposia and Invited Papers. East Lansing, MI: Philosophy of Science Association. Reprinted in Search for a Naturalistic Worldview, Volume II: Natural Science and Metaphysics. (Cambridge: Cambridge University Press, 1993), pp. 55-67.

Simon, C., Bužek, V., and Gisin, N. (2001). "No-signalling condition and quantum dynamics," Physical Review Letters, 87: 170405.

Tumulka, R. (2006). "A relativistic version of the Ghirardi-Rimini-Weber model," Journal of Statistical Physics, 125: 825-844.

Tumulka, Roderich. 2007. "The unromantic pictures of quantum theory," Journal of Physics A: Mathematical and Theoretical, 40: 3245-3273. 